\title{
Cardinal estimates involving the weak Lindelöf game
}

\author{
Leandro Aurichi ${ }^{1} \cdot$ Angelo Bella ${ }^{2}$. Santi Spadaro ${ }^{3}$
}

Received: 8 February 2021 / Accepted: 7 September 2021 / Published online: 27 September 2021

(c) The Author(s) 2021

\begin{abstract}
We show that if $X$ is a first-countable Urysohn space where player II has a winning strategy in the game $G_{1}^{\omega_{1}}\left(\mathcal{O}, \mathcal{O}_{D}\right)$ (the weak Lindelöf game of length $\left.\omega_{1}\right)$ then $X$ has cardinality at most continuum. This may be considered a partial answer to an old question of Bell, Ginsburg and Woods. It is also the best result of this kind since there are Hausdorff first-countable spaces of arbitrarily large cardinality where player II has a winning strategy even in the weak Lindelöf game of countable length. We also tackle the problem of finding a bound on the cardinality of a first-countable space where player II has a winning strategy in the game $G_{\text {fin }}^{\omega_{1}}\left(\mathcal{O}, \mathcal{O}_{D}\right)$, providing some partial answers to it. We finish by constructing an example of a compact space where player II does not have a winning strategy in the weak Lindelöf game of length $\omega_{1}$.
\end{abstract}

Keywords Cardinality bounds · Cardinal invariants · First-countable $\cdot$ Lindelöf · Weakly Lindelöf · Topological game

Mathematics Subject Classification 54A25 - 54D20 · 54D10

\section{Introduction}

All spaces are assumed to be Hausdorff. The letter $\kappa$ denotes an infinite cardinal throughout the paper.

One of the most intriguing open questions regarding cardinal functions in topology was asked by Bell, Ginsburg and Woods [1] about 40 years ago.

\section{Santi Spadaro}

santidomenico.spadaro@unipa.it

Leandro Aurichi

aurichi@icmc.usp.br

Angelo Bella

bella@dmi.unict.it

1 Instituto de Ciencias Matematicas e de Computacao (ICMC-USP), Universidade de Sao Paulo, Avenida Trabalhador Sao-Carlense, 400, Centro, Sao Carlos, SP 13566-590, Brazil

2 Dipartimento di Matematica e Informatica, Università di Catania, viale A. Doria 6, 95125 Catania, Italy

3 Dipartimento di Ingegneria, Università di Palermo, Viale delle Scienze, Ed. 8, 90128 Palermo, Italy 
Question 1.1 Let $X$ be a regular space. Is it true that $|X| \leq 2^{w L(X) \cdot \chi(X)}$ ?

Here $\chi(X)$ denotes the character of $X$ and $w L(X)$ denotes the weak Lindelöf number of $X$, that is the minimum infinite cardinal $\kappa$ such that, for every open cover $\mathcal{U}$ of $X$, there is a subcollection $\mathcal{V}$ of $\mathcal{U}$ of cardinality at most $\kappa$ such that $X \subset \overline{\cup \mathcal{V}}$.

The authors of [1] proved that the answer to Question 1.1 is positive for normal spaces, thus generalizing Arhangel'skii's theorem that every compact first-countable Hausdorff space has cardinality at most continuum [13], and constructed an example of a first-countable Hausdorff space $X$ such that $w L(X)=\aleph_{0}$ and $X$ can have arbitrary large cardinality.

Bell, Ginsburg and Woods's main motivation to pose Question 1.1 was to find, at least within the realm of regular spaces, a common generalization to Arhangel'skii's Theorem and the Hajnal-Juhász's inequality stating that every first-countable space with the countable chain condition has cardinality at most continuum. Such a generalization was eventually found, even without assuming regularity, by the second and third author in [3].

The main reason why Question 1.1 is so hard to solve is that, unlike the Lindelöf number, the weak Lindelöf number is not inherited by closed sets. To get around this difficulty, Arhangel'skii proposed a relative version of the weak Lindelöf number: the weak Lindelöf number for closed sets $\left(w L_{c}(X)\right)$ of $X$ is defined as the least infinite cardinal $\kappa$ such that for every closed set $F \subset X$ and for every open family $\mathcal{U}$ covering $F$ there is a subfamily $\mathcal{V} \subset \mathcal{U}$ having cardinality at most $\kappa$ such that $F \subset \overline{\cup \mathcal{V}}$. Arhangel'skii proved that $|X| \leq$ $2^{\chi(X) \cdot w L_{c}(X)}$, for every regular space $X$ and asked the following natural question.

Question 1.2 Is $|X| \leq 2^{w L_{c}(X) \cdot \chi(X)}$ for every Hausdorff space $X$ ?

A fair amount of progress has been done on Questions 1.1 and 1.2 since 1978. For example, in [4] Alas proved, among other things, that $|X| \leq 2^{w L_{c}(X) \cdot \chi(X)}$ for every Urysohn space $X$. In [5] Bella and Carlson proved that Question 1.1 has a positive answer for locally compact spaces and in [6] Gotchev proved that Question 1.1 has a positive answer for spaces with a regular $G_{\delta}$ diagonal.

In [7], the second and the third author proposed an attack to Bell, Ginsburg and Woods's question using the scheme of topological games.

Definition 1 Let $X$ be a topological space. We define a game $G_{1}^{\kappa}\left(\mathcal{O}, \mathcal{O}_{D}\right)$ (respectively, $\left.G_{\text {fin }}^{\kappa}\left(\mathcal{O}, \mathcal{O}_{D}\right)\right)$ as follows: at inning $\alpha<\kappa$, player I plays an open cover $\mathcal{U}_{\alpha}$ of $X$ and player II plays an open set $U_{\alpha} \in \mathcal{U}_{\alpha}$ (respectively a finite subcollection $\mathcal{F}_{\alpha} \subset \mathcal{U}_{\alpha}$ ). Player II wins if the collection $\left\{U_{\alpha}: \alpha<\kappa\right\}$ has a dense union in $X$ (respectively, if the collection $\bigcup\left\{\mathcal{F}_{\alpha}: \alpha<\kappa\right\}$ has a dense union in $X$ ).

Recall that a strategy for Player II in $G_{\text {fin }}^{\kappa}\left(\mathcal{O}, \mathcal{O}_{D}\right)$ is a function $\sigma: \mathcal{O}^{<\kappa} \rightarrow[\cup \mathcal{O}]^{<\omega}$ such that for any $s \in \mathcal{O}^{\alpha+1}$ we have $\sigma(s) \subset s(\alpha)$. The strategy $\sigma$ is a winning strategy if for any function $f \in \mathcal{O}^{\kappa}$ the set $\bigcup\{\bigcup \sigma(f\lceil\alpha): \alpha<\kappa\}$ is dense in $X$.

It is clear that if player II has a winning strategy in $G_{1}^{\kappa}\left(\mathcal{O}, \mathcal{O}_{D}\right)$ on $X$ then player II also has a winning strategy in $G_{\text {fin }}^{\kappa}\left(\mathcal{O}, \mathcal{O}_{D}\right)$ on $X$ and that in turn implies that $w L(X) \leq \kappa$. The authors of [7] proved that if $X$ is a first-countable regular space where player II has a winning strategy in the game $G_{1}^{\omega_{1}}\left(\mathcal{O}, \mathcal{O}_{D}\right)$ then $|X| \leq 2^{\aleph_{0}}$.

In the first section of our paper we will prove that regularity can be relaxed to the Urysohn separation property in the above result and show that this is the best possible result of this kind, since there are Hausdorff spaces of arbitrarily large cardinality where player II even has a winning strategy in $G_{1}^{\omega}\left(\mathcal{O}, \mathcal{O}_{D}\right)$.

It is natural to ask whether a winning strategy for player II in $G_{f i n}^{\omega_{1}}\left(\mathcal{O}, \mathcal{O}_{D}\right)$ is enough to bound the cardinality of a first-countable regular space. That's open, but we prove that 
assuming that the space is Tychonoff and that the winning strategy for player II is monotone, then the bound is the continuum.

A question from [7] asks whether there is a first-countable regular space where player II does not have a winning strategy in $G_{1}^{\omega_{1}}\left(\mathcal{O}, \mathcal{O}_{D}\right)$. It turned out that no example of a weakly Lindelöf space where player II does not have a winning strategy in $G_{1}^{\omega_{1}}\left(\mathcal{O}, \mathcal{O}_{D}\right)$ is known. In the final section of our paper we will construct an example of a compact space where even player I has a winning strategy in $G_{1}^{\omega_{1}}\left(\mathcal{O}, \mathcal{O}_{D}\right)$.

As usual the notation $\beta X$ denotes the Čech-Stone compactification of a Tychonoff space $X$. Undefined notions may be found in [8], although our notation regarding cardinal functions mostly follows [9]. To streamline proofs we sometimes make use of elementary substructures of $(H(\theta), \epsilon)$. The reader who is not familiar with this technique is referred to the first few sections of [10].

\section{The weak Lindelöf game and cardinality}

Two games $G$ and $G^{\prime}$ are said to be dual if (1) player I has a winning strategy in $G$ if and only if player II has a winning strategy in $G^{\prime}$ and (2) player II has a winning strategy in $G$ if and only if player I has a winning strategy in $G^{\prime}$. In [11] we proved that $G_{1}^{\kappa}\left(\mathcal{O}, \mathcal{O}_{D}\right)$ is the dual of the following game.

Definition 2 Let $G_{o}^{p}(\kappa)$ denote the game in $\kappa$ many innings where, at inning $\alpha<\kappa$ player I picks a point $x_{\alpha}$ and player II chooses an open neighbourhood $U_{\alpha}$ of $x_{\alpha}$. Player I wins if $\left\{U_{\alpha}: \alpha<\kappa\right\}$ has a dense union in $X$.

Recall that a space $X$ is Urysohn if for every pair of distinct points $x, y \in X$ there are open neighbourhoods $U$ of $x$ and $V$ of $y$ such that $\bar{U} \cap \bar{V}=\emptyset$. Given a set $A \subset X$, we say that $x$ is a $\theta$-limit point of $A$ if $\bar{V} \cap A \neq \emptyset$, for every open neighbourhood $V$ of $x$. We define the $\theta$-closure of $A\left(C l_{\theta}(A)\right)$ as the set of all $\theta$-limit points of $A$. We say that $A \subset X$ is $\theta$-closed if $C l_{\theta}(A)=A$ and that $A \subset X$ is $\theta$-dense in $X$ if $C l_{\theta}(A)=X$.

Theorem 3 Let $(X, \tau)$ be a Urysohn space such that $\chi(X)<\kappa$ and player II has a winning strategy in the game $G_{1}^{\kappa}\left(\mathcal{O}, \mathcal{O}_{D}\right)$. Then $|X| \leq 2^{<\kappa}$.

Proof Using the duality between $G_{1}^{\kappa}\left(\mathcal{O}, \mathcal{O}_{D}\right)$ and $G_{o}^{p}(\kappa)$ we can fix a winning strategy $\sigma$ for player I in $G_{o}^{p}(\kappa)$. Let $\mu$ be a large enough regular cardinal, let $M$ be a $<\kappa$-closed elementary submodel of $H(\mu)$ of cardinality $2^{<\kappa}$ such that $X, \tau, \sigma \in M$ and $\kappa+1 \subset M$.

Claim $1 X \cap M$ is $\theta$-closed.

Proof of Claim 1 Let $x \in C l_{\theta}(X \cap M)$ and let $\left\{U_{\alpha}: \alpha<\mu\right\}$ be a local base at $x$, where $\mu<\kappa$. For every $\alpha<\mu$ choose a point $x_{\alpha} \in \overline{U_{\alpha}} \cap M$ and let $S=\left\{x_{\alpha}: \alpha<\mu\right\}$. Note that $x \in C l_{\theta}(S)$ and hence $x \in C l_{\theta}\left(S \cap \overline{U_{\alpha}}\right)$, for every $\alpha<\mu$. Moreover, since $M$ is a $<\kappa$-closed elementary submodel, $S \cap \overline{U_{\alpha}} \in M$ and hence $C l_{\theta}\left(S \cap \overline{U_{\alpha}}\right) \in M$.

The fact that $X$ is a Urysohn space implies that $\bigcap\left\{C l_{\theta}\left(\overline{U_{\alpha}} \cap S\right): \alpha<\mu\right\}=\{x\}$. Now, using the $<\kappa$-closedness of $M$ again, we gather that $\{x\}=\bigcap\left\{C l_{\theta}\left(\overline{U_{\alpha}} \cap S\right): \alpha<\mu\right\} \in M$. Hence $x \in X \cap M$, which proves that $X \cap M$ is $\theta$-closed. $\triangle$

Claim $2 X \cap M$ is $\theta$-dense. 
Proof of Claim 2 If that weren't true, there would exist an open set $V$ such that $\bar{V} \cap X \cap M=\emptyset$. In the first inning, playing according to the strategy $\sigma$, player I picks a point $x_{0}=\sigma(\emptyset) \in M$. Since $x_{0} \in M$ and $\chi(X)<\kappa$ we can choose a local base $\left\{V_{\alpha}^{\prime}: \alpha<\mu\right\} \in M$ for $x_{0}$, where $\mu<\kappa$. Since $\kappa+1 \subset M$ we have $\left\{V_{\alpha}^{\prime}: \alpha<\mu\right\} \subset M$. Since $x_{0} \notin \bar{V}$ we can choose $\alpha_{0}<\mu$ such that $V_{\alpha_{0}}^{\prime} \cap V=\emptyset$. Let player II play $V_{0}=V_{\alpha_{0}}^{\prime}$ in the first inning.

Let now $\beta<\kappa$ and suppose that, at inning $\alpha<\beta$, player II played an open set $U_{\beta} \in M$ such that $U_{\beta} \cap V=\emptyset$. Since $M$ is $<\kappa$-closed, player I's choice at inning $\beta$, i.e. $x_{\beta}=$ $\sigma\left(\left(U_{\alpha}: \alpha<\beta\right)\right)$ is an element of $X \cap M$. Reasoning as in the previous paragraph, player II can play an open set $V_{\beta} \in M$ such that $V_{\beta} \cap V=\emptyset$.

At the end of the game, since $\sigma$ is a winning strategy for player I in $G_{o}^{p}(\kappa)$ we have that $\bigcup\left\{V_{\alpha}: \alpha<\kappa\right\}$ is dense in $X$, but that contradicts the fact that $V \cap V_{\alpha}=\emptyset$, for every $\alpha<\kappa$. $\triangle$

Since $X \cap M$ is both $\theta$-closed and $\theta$-dense, we have $X \cap M=X$ and hence $|X|=$ $|X \cap M| \leq 2^{<\kappa}$, as we wanted.

Corollary 4 Let $X$ be a Urysohn first-countable space where player II has a winning strategy in $G_{1}^{\omega_{1}}\left(\mathcal{O}, \mathcal{O}_{D}\right)$. Then $|X| \leq 2^{\aleph_{0}}$.

The following example shows that Theorem 3 is sharp.

Example 5 A first-countable Hausdorff space of arbitrarily large cardinality where player II has a winning strategy in $G_{1}^{\omega}\left(\mathcal{O}, \mathcal{O}_{D}\right)$.

Proof Let $D_{1}$ be a countable dense subset of the reals and let $D_{2}=\mathbb{R} \backslash D_{1}$ be its complement. Define a topology $\tau$ on $X=D_{1} \cup\left(D_{2} \times \kappa\right)$ as follows: a basic neighbourhood of the point $(x, \alpha) \in D_{2} \times \kappa$ has the form $\left(U \cap D_{2}\right) \times\{\alpha\}$, where $U$ is an open neighbourhood of $x$ in the euclidean topology. A basic neighbourhood of the point $x \in D_{1}$ has the form $\left(U \cap D_{1}\right) \cup\left(\left(U \cap D_{2}\right) \times \kappa\right)$, where $U$ is a euclidean open neighbourhood of $x$. It is easy to see that $X$ is a Hausdorff first-countable space.

We now describe a winning strategy for player II in $G_{1}^{\omega}\left(\mathcal{O}, \mathcal{O}_{D}\right)$. Let $\left\{x_{n}: n<\omega\right\}$ be an enumeration of $D_{1}$. At inning $n<\omega$, player I chooses an open cover $\mathcal{O}_{n}$ for $X$. Let player II pick an open set $O_{n} \in \mathcal{O}_{n}$ such that $x_{n} \in O_{n}$. It was proved in [1] that every open set containing $D_{1}$ is dense in $X$, therefore $\bigcup\left\{O_{n}: n<\omega\right\}$ is dense in $X$.

However, if we assume the space to be countably compact we can trade the Urysohn property for the Hausdorff property.

Theorem 6 Let $(X, \tau)$ be a first-countable countably compact Hausdorff space where player II has a winning strategy in $G_{1}^{\omega_{1}}\left(\mathcal{O}, \mathcal{O}_{D}\right)$. Then $|X| \leq 2^{\aleph_{0}}$.

Proof Let $\sigma$ be a winning strategy for player I in $G_{o}^{p}\left(\omega_{1}\right)$. Let $\mu$ be a large enough regular cardinal and let $M$ be an $\omega$-closed elementary submodel of $H(\mu)$ of cardinality $2^{\omega}$ such that $X, \tau, \sigma \in M$.

Claim 1. $X \cap M$ is countably compact.

Proof of Claim 1 Let $C$ be a countable subset of $X \cap M$. Since $X$ is countably compact, the set $C$ has an accumulation point in $X$. Since $M$ is countably closed, we have $C \in M$. But then elementarity implies that $C$ has an accumulation point in $X \cap M$ and hence we're done. $\triangle$ 
We will prove that $X \cap M=X$. Suppose that this is not the case, and let $p \in X \backslash M$. Claim 2. There is an open collection $\mathcal{U} \subset M$ such that $X \cap M \subset \cup \mathcal{U}$ and $p \notin \overline{\cup \mathcal{U}}$.

Proof of Claim 2 Fix a countable decreasing local base $\left\{V_{n}: n<\omega\right\}$ at $p$. For every $x \in X \cap M$ fix a countable decreasing local base $\left\{U_{n}^{x}: n<\omega\right\}$ at $x$. Without loss of generality we can assume $\left\{U_{n}^{x}: n<\omega\right\} \in M$ and hence $\omega \subset M$ implies that $\left\{U_{n}^{x}: n<\omega\right\} \subset M$. By the Hausdorff property of $X$ there must be an integer $n(x)<\omega$ such that $U_{n(x)}^{x} \cap V_{n(x)}=\emptyset$. Then $\left\{U_{n(x)}^{x}: x \in X \cap M\right\}$ is an open cover of $X \cap M$. Let $O_{n}=\bigcup\left\{U_{n(x)}^{x}: n(x)=n\right\}$. Then $\left\{O_{n}: n<\omega\right\}$ is also an open cover of $X \cap M$ and hence, since $X \cap M$ is countably compact, there is an integer $n_{0}<\omega$ such that $X \cap M \subset \bigcup\left\{O_{n}: n \leq n_{0}\right\}$. Then $\mathcal{U}=\left\{U_{n(x)}^{x}\right.$ : $\left.n(x) \leq n_{0}\right\}$ is the open collection required by the Claim, since $\mathcal{U} \subset M, \mathcal{U}$ covers $X \cap M$ and $V_{n_{0}} \cap \bigcup \mathcal{U}=\emptyset . \triangle$

We will now play a game of $G_{o}^{p}\left(\omega_{1}\right)$ where player I plays according to the winning strategy $\sigma$. In the first inning player I picks a point $x_{0}=\sigma(\emptyset) \in M$. Player two then selects an open set $U_{0} \in \mathcal{U}$ such that $x_{0} \in U_{0}$.

Let now $\beta<\omega_{1}$ and suppose that, at inning $\alpha<\beta$, player II picked the open set $U_{\alpha} \in \mathcal{U} \subset M$. Since $M$ is $\omega$-closed, player I's choice at inning $\beta$, i.e. $x_{\beta}=\sigma\left(\left(U_{\alpha}: \alpha<\beta\right)\right)$ in an element of $X \cap M$. Let player II select an open set $U_{\beta} \in \mathcal{U}$ such that $x_{\beta} \in U_{\beta}$.

At the end of the game, since $\sigma$ is a winning strategy for player I in $G_{o}^{p}\left(\omega_{1}\right)$ we have that $\bigcup\left\{U_{\alpha}: \alpha<\omega_{1}\right\}$ is dense in $X$, but that contradicts the fact that $p \notin \overline{\cup \mathcal{U}}$.

Question 2.1 Let X be a Urysohn (or even regular) first-countable space such that player II has a winning strategy in $G_{\text {fin }}^{\omega_{1}}\left(\mathcal{O}, \mathcal{O}_{D}\right)$ (or even in the corresponding game of countable length). Is it true that $|X| \leq 2^{\aleph_{0}}$ ?

While in Arhangel'skii's theorem $|X| \leq 2^{\chi(X) \cdot L(X)}$ it is possible to replace the character with the product of the tightness and the pseudocharacter, that is not the case with Theorem 3 . Indeed, Bell, Ginsburg and Woods constructed in [1] an example of a regular space with countable tightness and countable pseudocharacter $X$, containing a countable set $D$ with the property that, if $U \subset X$ is an open set containing $D$, then $U$ is dense in $X$. The argument from Example 5 then shows that player II has a winning strategy in $G_{1}^{\omega}\left(\mathcal{O}, \mathcal{O}_{D}\right)$ on $X$.

However, we were not able to answer the following question.

Question 2.2 Is there a bound on the cardinality of normal spaces of countable tightness and countable pseudocharacter where player II has a winning strategy in $G_{1}^{\omega}\left(\mathcal{O}, \mathcal{O}_{D}\right)$ ?

\section{Allowing the second player to choose finitely many open sets}

Theorem 3 suggests a natural question.

Question 3.1 Let $X$ be a first-countable regular space where player II has a winning strategy in $G_{\text {fin }}^{\omega_{1}}\left(\mathcal{O}, \mathcal{O}_{D}\right)$. Is $|X| \leq 2^{\aleph_{0}}$ ?

We will offer some partial answers to the above question. To start with, we will consider a game which appears to be close to $G_{\text {fin }}^{\omega_{1}}\left(\mathcal{O}, \mathcal{O}_{D}\right)$.

Recall the following definition.

Definition 7 An open cover $\mathcal{O}$ for a topological space $X$ is said to be a $K$-cover if, for every compact set $K \subset X$ there is an open set $O \in \mathcal{O}$ such that $K \subset O$. We will denote the set of all open $K$-covers of $X$ by $\mathcal{O}_{K}$. 
The games $G_{\text {fin }}^{\kappa}(\mathcal{O}, \mathcal{O})$ and $G_{1}^{\kappa}\left(\mathcal{O}_{K}, \mathcal{O}\right)$ are equivalent (for regular spaces), so it is natural to ask the following question:

Question 3.2 Are the games $G_{\text {fin }}^{\kappa}\left(\mathcal{O}, \mathcal{O}_{D}\right)$ and $G_{1}^{\kappa}\left(\mathcal{O}_{K}, \mathcal{O}_{D}\right)$ equivalent?

Let $G_{o}^{k}(\kappa)$ be the game in $\kappa$ many innings where at inning $\alpha<\kappa$ player I plays a compact set $K_{\alpha} \subset X$, player II plays an open set $U_{\alpha} \supset K_{\alpha}$ and player I wins if $\bigcup\left\{U_{\alpha}: \alpha<\kappa\right\}$ is dense in $X$. The proof of the following lemma is a simple modification of the proof of Theorem 3.8 from [11].

Lemma 8 The games $G_{1}^{\kappa}\left(\mathcal{O}_{K}, \mathcal{O}_{D}\right)$ and $G_{o}^{k}(\kappa)$ are dual.

Theorem 9 Let $X$ be a regular first-countable space such that player II has a winning strategy in the game $G_{1}^{\omega_{1}}\left(\mathcal{O}_{K}, \mathcal{O}_{D}\right)$. Then $|X| \leq 2^{\aleph_{0}}$.

Proof By Lemma 8 we can fix a winning strategy $\sigma$ for player I in $G_{o}^{k}(\kappa)$. Also, since $X$ is first-countable, by Arhangel'skii's Theorem every compact subset of $X$ has cardinality at most continuum. Denote by $\mathcal{K}$ the set of all compact subsets of $X$. It turns out that every $K \in \mathcal{K}$ has an outer base of size continuum (i.e., a family $\mathcal{B}$ of open sets such that given an open set $V \supset K$, there is a member $B \in \mathcal{B}$ such that $K \subset B \subset V$ ). So, for every compact subset $K \subset X$, let $\mathcal{B}_{K}$ be an outer base for $K$. Now, fix $M$ a countably closed elementary submodel containing everything we need ( $\sigma, X$, and etc.) and such that $|M|=2^{\omega}$. Our goal is to show that $X \subset M$. Since $X$ is first countable, $X \cap M$ is closed in $X$. If we can prove that $X \cap M$ is dense in $X$ we are done.

Claim. $X \cap M$ is dense in $M$.

Proof of Claim Suppose that $X \cap M$ is not dense. Since $X$ is regular, there is a non-empty closed set $F$ such that $F \cap(X \cap M)=\emptyset$. Fix $K \in \mathcal{K} \cap M$. Note that $K \subset M$ (since $K$ has cardinality at most continuum) and hence $F \cap K=\emptyset$. Also we can assume that the outer base $\mathcal{B}_{K}$ belongs to $M$ and since $\left|\mathcal{B}_{K}\right| \leq 2^{\aleph_{0}}$ we have that $\mathcal{B}_{K} \subset M$.

We can now play a game of $G_{o}^{k}\left(\omega_{1}\right)$ where player I uses the winning strategy $\sigma$ and player II picks an open set $V \in M$ such that $V \cap F=\emptyset$. The second player would win such a game and that is a contradiction. $\triangle$

We will say that a strategy for player II is monotone if whenever $\mathcal{C}_{1}$ refines $\mathcal{C}_{2}$ then $\bigcup \sigma\left(s \frown \mathcal{C}_{1}\right) \subset \bigcup \sigma\left(s \frown \mathcal{C}_{2}\right)$ for every $s \in \mathcal{O}^{<\omega_{1}}$. We will say that a set $K \subset X$ is good if there is an $s \in \mathcal{O}^{<\omega_{1}}$ such that $K=\bigcap\left\{C l_{\beta X}(\bigcup \sigma(s \frown \mathcal{C})): \mathcal{C} \in \mathcal{O}\right\}$. It is clear that every good set is compact.

Lemma 10 Let $\sigma$ be a monotone strategy for player II in $G_{\text {fin }}^{\omega_{1}}\left(\mathcal{O}, \mathcal{O}_{D}\right)$ over the space X. If $K=\bigcap\left\{C l_{\beta X}(\bigcup \sigma(s \sim \mathcal{C})): \mathcal{C} \in \mathcal{O}\right\}$, for some $s \in \mathcal{O}^{<\omega_{1}}$ and $K$ is contained in an open set $V \subset X$, then there is some $\mathcal{C} \in \mathcal{O}$ such that $\overline{\sigma(s \frown \mathcal{C})} \subset V$.

Proof Let $W$ be an open subset of $\beta X$ such that $W \cap X=V$. By compactness there are $\mathcal{C}_{0}, \ldots \mathcal{C}_{n} \in \mathcal{O}$ such that:

$$
\bigcap_{i \leq n} C l_{\beta X}\left(\bigcup \sigma\left(s \frown \mathcal{C}_{i}\right)\right) \subset W
$$

From this it follows that $\bigcap_{i \leq n} \overline{\cup \sigma\left(s \frown \mathcal{C}_{i}\right)} \subset V$. Now, since $\sigma$ is monotone it suffices to take an open cover $\mathcal{C}$ which refines $\mathcal{C}_{1}, \ldots \mathcal{C}_{n}$. 
Lemma 11 Let $X$ be a sequential space such that $\chi(X) \leq 2^{\omega}$ and let $K$ be a compact subset of $X$. Then there is a sequence $\left\{V_{\alpha}: \alpha<2^{\omega}\right\}$ of open subsets of $X$ which forms an outer base for $K$ in $X$.

Proof First note that each compact subset of $X$ has cardinality at most continuum (see, for example, [12], Theorem 3.2). Hence $|K| \leq 2^{\omega}$. For every $x \in K$, let $\mathcal{U}_{x}$ be a local base for $x$ having cardinality at most continuum. Let $\mathcal{B}=\left\{\bigcup \mathcal{W}: \mathcal{W} \in\left[\bigcup\left\{\mathcal{U}_{x}: x \in K\right\}\right]^{<\omega}, K \subset\right.$ $\cup \mathcal{W}\}$. Note that $\mathcal{B}$ is an outer base for $K$ in $X$ having cardinality at most continuum.

Let $S$ be a subset of a topological space $X$. We say that a collection $\mathcal{D}$ of subsets of $X$ is a local network for $S$ in $X$ provided that for every open set $V \subset X$ such that $S \subset V$ there is a set $D \in \mathcal{D}$ such that $S \subset D \subset V$.

From the previous two lemmas we immediately get the following:

Lemma 12 Let $X$ be a sequential space such that $\chi(X) \leq 2^{\omega}$ and let $\sigma$ be a monotone strategy for player II in $G_{\text {fin }}^{\omega_{1}}\left(\mathcal{O}, \mathcal{O}_{D}\right)$. If $K=\bigcap\left\{C l_{\beta X}(\bigcup \sigma(s \frown \mathcal{C})): \mathcal{C} \in \mathcal{O}\right\}$, for some $s \in \mathcal{O}^{<\omega_{1}}$, then there is $\mathcal{O}^{\prime} \subset \mathcal{O}$ such that $\left|\mathcal{O}^{\prime}\right| \leq 2^{\omega}$ and $\left\{\overline{\bigcup \sigma(s \frown \mathcal{C})}: \mathcal{C} \in \mathcal{O}^{\prime}\right\}$ is a local network for $K$ in $X$.

Theorem 13 Let $X$ be a sequential space with $\chi(X) \leq 2^{\omega}$ such that player II has a monotone winning strategy $\sigma$ for the game $G_{\text {fin }}^{\omega_{1}}\left(\mathcal{O}, \mathcal{O}_{D}\right)$. Then $|X| \leq 2^{\omega}$.

Proof Let $\theta$ be a large enough regular cardinal and let $M$ be an elementary submodel of $H(\theta)$ such that $|M| \leq 2^{\omega}, X, \sigma \in M$ and $[M]^{\omega} \subset M$. Let $\mathcal{K}=\{K \subset X: K \in M$ and $K$ is good $\}$. Since $X$ is sequential and every compact subset of $X$ has cardinality continuum, it is enough to show that $\bigcup \mathcal{K}$ is dense in $X$.

Assuming the contrary, there is a non-empty open set $H$ such that $\bar{H} \subset X \backslash \bigcup \mathcal{K}$. Let $K_{0}=\bigcap\left\{C l_{\beta X}(\bigcup \sigma(\mathcal{C})): \mathcal{C} \in \mathcal{O}\right\}$. Note that $K_{0} \in M$ and hence $K_{0} \in \mathcal{K}$. By Lemma 12 and elementarity we can find a subset $\mathcal{O}^{\prime} \subset \mathcal{O}$ such that $\left|\mathcal{O}^{\prime}\right| \leq 2^{\omega}, \mathcal{O}^{\prime} \in M$ and $\mathcal{D}=\{\overline{\sigma(\mathcal{C})}$ : $\left.\mathcal{C} \in \mathcal{O}^{\prime}\right\}$ is a local network for $K_{0}$. Thus there is $\mathcal{C}_{0} \in \mathcal{O}^{\prime}$ such that $\overline{\sigma\left(\mathcal{C}_{0}\right)} \subset X \backslash \bar{H}$. Since $\mathfrak{c}+1 \subset M$ we actually have $\mathcal{D} \subset M$ and hence $\bigcup \sigma\left(\mathcal{C}_{0}\right) \in M$.

We now proceed by induction. Assume you have already defined open covers $\left\{\mathcal{C}_{\alpha}: \alpha<\right.$ $\beta\} \subset M$ and define $s: \beta \rightarrow \mathcal{O}$ by letting $s(\alpha)=\mathcal{C}_{\alpha}$, for all $\alpha<\beta$. Since $M$ is $\omega$-closed, we have $s \in M$. Therefore $K_{\beta}=\bigcap\{\overline{\cup(s \frown \mathcal{C})}: \mathcal{C} \in \mathcal{O}\} \in M$ and hence $K_{\beta} \in \mathcal{K}$. Then, reasoning as before, we obtain $C_{\beta} \in \mathcal{O} \cap M$ such that $H \cap \sigma\left(s \frown \mathcal{C}_{\beta}\right)=\emptyset$.

This defines a play of $G_{f i n}^{\omega_{1}}\left(\mathcal{O}, \mathcal{O}_{D}\right)$ where player I wins, in contradiction with the fact that $\sigma$ is a winning strategy for player II.

\section{A compact space where player I has a winning strategy in $G_{1}^{\omega_{1}}\left(\mathcal{O}, \mathcal{O}_{D}\right)$}

In [7], the second and the third author asked whether there is an example of a weakly Lindelöf first-countable space $X$ where player II does not have a winning strategy in $G_{1}^{\omega_{1}}\left(\mathcal{O}, \mathcal{O}_{D}\right)$. This question remains open, and if no such example existed, the approach of [7] would lead to a complete solution to Bell, Ginsburg and Woods's question.

It turned out that the above question was open even without the first-countability assumption. We now exhibit an example of a (non-first countable) compact space where it even holds that player I has a winning strategy in $G_{1}^{\omega_{1}}\left(\mathcal{O}, \mathcal{O}_{D}\right)$. By modifying an argument of Dias and Tall [13], we will show that the space $2^{\omega_{2}}$ with the lexicographic order topology is such an example. 
Given $f, g \in 2^{\omega_{2}}$, let $\Delta(f, g)=\min \left\{\alpha<\omega_{2}: f(\alpha) \neq g(\alpha)\right\}$. We define an order on $2^{\omega_{2}}$ by saying that $f<g$ if and only if $f(\Delta(f, g))<g(\Delta(f, g))$.

Lemma 14 Let $f, g \in 2^{\omega_{2}}$ be two functions such that $f<g$. Then the open interval $(f, g)$ is empty if and only if for every $\alpha>\Delta(f, g)$ we have $f(\alpha)>g(\alpha)$.

Proof Suppose there is $\alpha>\Delta(f, g)$ such that $f(\alpha) \leq g(\alpha)$. Then one of the following cases can occur:

(1) If $f(\alpha)=0=g(\alpha)$, then let $h \in 2^{\omega_{2}}$ be any function such that $h(\alpha)=1$ and $h(\gamma)=$ $f(\gamma)$, for every $\gamma<\alpha$. We have $f<h<g$.

(2) If $f(\alpha)=1=g(\alpha)$, then let $h \in 2^{\omega_{2}}$ be any function such that $h(\alpha)=0$ and $h(\gamma)=$ $g(\gamma)$, for every $\gamma<\alpha$. We have $f<h<g$.

(3) If $f(\alpha)=0$ and $g(\alpha)=1$, let $h$ be any function such that $h(\gamma)=f(\gamma)$, for every $\gamma<\alpha$ and $h(\alpha)=1$. We have $f<h<g$.

In any case, $h \in(f, g)$ and hence the interval $(f, g)$ is not empty.

Lemma 15 Let $f \in 2^{\omega_{2}}$ and let $A \subset 2^{\omega_{2}}$ be a set of cardinality $\aleph_{1}$.

(1) If $f=\sup A$ and $A$ has no greatest element, then the set $\left\{\beta<\omega_{2}: f(\beta)=1\right\}$ has cardinality $\leq \aleph_{1}$.

(2) If $g=\inf A$ and $A$ has no least element, then the $\operatorname{set}\left\{\beta<\omega_{2}: g(\beta)=0\right\}$ has cardinality $\leq \aleph_{1}$.

Proof Assume $A$ has no greatest element. Then $f \notin A$. Let $\delta=\sup \{\Delta(h, f): h \in A\}+1<$ $\omega_{2}$. We claim that $f(\alpha)=0$, for every $\alpha>\delta$. Indeed, suppose by contradiction that there is an ordinal $\beta>\delta$ such that $f(\beta)=1$. Let $y \in 2^{\omega_{2}}$ be defined as follows: $y(\gamma)=f(\gamma)$, for every $\gamma<\beta$ and $y(\gamma)=0$, for every $\gamma \geq \beta$. Then $y<f$ and $h<y$, for every $h \in A$, contradicting the fact that $f$ is the least upper bound of $A$. The proof of the second item is similar.

Recall that a space $X$ is called an almost $P_{\omega_{2}}$-space if for every $\leq \omega_{1}$-sized family $\left\{U_{\alpha}: \alpha<\omega_{1}\right\}$ open subsets of $X$ such that $\bigcap\left\{U_{\alpha}: \alpha<\omega_{1}\right\} \neq \emptyset$ we have $\operatorname{Int}\left(\bigcap\left\{U_{\alpha}: \alpha<\right.\right.$ $\left.\left.\omega_{1}\right\}\right) \neq \emptyset$.

Lemma 16 The space $2^{\omega_{2}}$ with the lexicographic order topology is an almost $P_{\omega_{2}}$-space.

Proof Let $\left\{U_{\alpha}: \alpha<\omega_{1}\right\}$ be an $\omega_{1}$-sequence of open sets whose intersection is non-empty and fix $h \in \bigcap\left\{U_{\alpha}: \alpha<\omega_{1}\right\}$. Then we can find open intervals $\left\{\left(f_{\alpha}, g_{\alpha}\right): \alpha<\omega_{1}\right\}$ such that $h \in\left(f_{\alpha}, g_{\alpha}\right) \subset U_{\alpha}$, for every $\alpha<\omega_{1}$. If we can prove that there is $r \in 2^{\omega_{2}}$ such that $r \neq h$ and $r \in \bigcap_{\alpha<\omega_{1}}\left(f_{\alpha}, g_{\alpha}\right)$ then we are done, because we'd have $(\min \{h, r\}, \max \{h, r\}) \subset$ $\bigcap_{\alpha<\omega_{1}}\left(f_{\alpha}, g_{\alpha}\right)$.

Suppose by contradiction that $\bigcap_{\alpha<\omega_{1}}\left(f_{\alpha}, g_{\alpha}\right)=\{h\}$ and let $f=\sup \left\{f_{\alpha}: \alpha<\omega_{1}\right\}$ and $g=\inf \left\{g_{\alpha}: \alpha<\omega_{1}\right\}$. We have $f \leq h \leq g$.

We can't have $f<h<g$, because that would imply both $(f, h)=\emptyset$ and $(h, g)=\emptyset$, but these can't happen at the same time because that would contradict Lemma 14. Also, we can't have $f=h=g$ because that would contradict Lemma 15. Therefore we must have either $f=h<g$, which implies $(h, g)=\emptyset$ and therefore, along with Lemma 14 it contradicts the second item of Lemma 15 or otherwise we must have $f<h=g$, which implies $(f, h)=\emptyset$ and therefore, along with Lemma 14 it contradicts the first item of Lemma 15. 
Theorem 17 Let $X$ be a compact almost $P_{\omega_{2}}$-space without isolated points. Then player I has a winning strategy in $G_{1}^{\omega_{1}}\left(\mathcal{O}, \mathcal{O}_{D}\right)$.

Proof The usual proof of the Čech-Pospišil Theorem shows that in a compact almost $P_{\omega_{2}}$ space there is a family of closed sets $\left\{F_{s}: s \in 2^{\leq \omega_{1}}\right\}$ such that:

(1) $\operatorname{Int}\left(F_{S}\right) \neq \emptyset$, for every $s \in 2^{\leq \omega_{1}}$.

(2) $s \subset t$ implies $F_{t} \subset F_{s}$.

(3) $F_{s} \frown(0) \cap F_{s} \frown(1)=\emptyset$.

(4) For $\beta \in \operatorname{Lim}\left(\omega_{1}+1\right)$ and $s \in 2^{\beta}, F_{s}=\bigcap_{\alpha<\beta} F_{s\lceil\alpha}$.

Let now $U_{s}=X \backslash F_{s}$, for every $s \in 2^{\leq \omega_{1}}$. We now describe a winning strategy for player I in $G_{1}^{\omega_{1}}\left(\mathcal{O}, \mathcal{O}_{D}\right)$. At the first inning, player I chooses the open cover $\left\{U_{(0)}, U_{(1)}\right\}$. Let now $\alpha<\omega_{1}$ and let $s \in 2^{\alpha}$ be a function such that player II's move at inning $i$ was $U_{s \uparrow i}$ for every $i<\alpha$. Then player I chooses the open cover $\left\{U_{s \frown(0)}, U_{s \frown(1)}\right\}$ at inning $\alpha$. At the end of the game, let $f \in 2^{\omega_{1}}$ be the function such that $U_{f\lceil\alpha}$ is the open set chosen by player II at inning $\alpha$. Then $\bigcup\left\{U_{f\lceil\alpha}: \alpha<\omega_{1}\right\}=U_{f}$ is not dense, since its complement, $F_{f}$ has non-empty interior.

Question 4.1 Is there a first-countable regular space $X$ such that player II does not have a winning strategy in $G_{1}^{\omega_{1}}\left(\mathcal{O}, \mathcal{O}_{D}\right)$ ?

Acknowledgements The first author is grateful to FAPESP for partial financial support through grant number 2019/22344-0. The second and the third author are grateful to INdAM-GNSAGA for partial financial support. The authors are grateful to the referee for their careful reading of the paper.

Funding Open access funding provided by Universitá degli Studi di Palermo within the CRUI-CARE Agreement.

Open Access This article is licensed under a Creative Commons Attribution 4.0 International License, which permits use, sharing, adaptation, distribution and reproduction in any medium or format, as long as you give appropriate credit to the original author(s) and the source, provide a link to the Creative Commons licence, and indicate if changes were made. The images or other third party material in this article are included in the article's Creative Commons licence, unless indicated otherwise in a credit line to the material. If material is not included in the article's Creative Commons licence and your intended use is not permitted by statutory regulation or exceeds the permitted use, you will need to obtain permission directly from the copyright holder. To view a copy of this licence, visit http://creativecommons.org/licenses/by/4.0/.

\section{References}

1. Bell, M., Ginsburg, J., Woods, G.: Cardinal inequalities for topological spaces involving the weak Lindelöf number. Pac. J. Math. 79, 37-45 (1978)

2. Arhangel'skiı̆, A.V.: On the cardinality of bicompacta satisfying the first axiom of countability. Sov. Math., Dokl. 10, 951-955 (1969); translation from Dokl. Akad. Nauk SSSR 187, 967-970 (1969)

3. Bella, A., Spadaro, S.: A common extension of Arhangel'skii's Theorem and the Hajnal-Juhász's inequality. Can. Math. Bull. 63, 197-203 (2020)

4. Alas, O.T.: More topological cardinal inequalities. Colloq. Mat. 65, 165-168 (1993)

5. Bella, A., Carlson, N.: On cardinality bounds involving the weak Lindelöf degree. Quaest. Math. 41, 99-113 (2018)

6. Gotchev, I.: Cardinalities of weakly Lindelöf spaces with regular $G_{\kappa}$ diagonals. Topol. Appl. 259, 80-89 (2019)

7. Bella, A., Spadaro, S.: Infinite games and cardinal properties of topological spaces. Houst. J. Math. 41, 1063-1077 (2015)

8. Engelking, R.: General topology. Heldermann-Verlag, Berlin (1989)

9. Juhász, I.: Cardinal functions in topology—ten years later, p. 123. Mathematisch Centrum, Amsterdam (1980) 
10. Dow, A.: An introduction to applications of elementary submodels to topology. Topol. Proc. 13(1), 17-72 (1988)

11. Aurichi, L., Spadaro, S., Zdomskyy, L.: Selective versions of chain condition-type properties. Acta Math. Hung. 148, 1-16 (2016)

12. Hodel, R.E.: Arhangel'skii's solution to Alexandroff's problem: a survey. Topol. Appl. 153, 2199-2217 (2006)

13. Dias, R.R., Tall, F.: Indestructibility of compact spaces. Topol. Appl. 160, 2411-2426 (2013)

Publisher's Note Springer Nature remains neutral with regard to jurisdictional claims in published maps and institutional affiliations. 